\title{
Possible long-term endocrine-metabolic complications in COVID-19: lesson from the SARS model
}

\author{
Laura M. Mongioi ${ }^{1} \cdot$ Federica Barbagallo ${ }^{1} \cdot$ Rosita A. Condorelli $^{1} \cdot$ Rossella Cannarella $^{1} \cdot$ Antonio Aversa $^{2}$. \\ Sandro La Vignera $\mathbb{D}^{1} \cdot$ Aldo E. Calogero ${ }^{1}$
}

Received: 29 April 2020 / Accepted: 12 May 2020 / Published online: 2 June 2020

(c) Springer Science+Business Media, LLC, part of Springer Nature 2020

\begin{abstract}
The outbreak of coronavirus disease 2019 (COVID-19), caused by severe acute respiratory syndrome coronavirus 2 (SARS$\mathrm{CoV}-2$ ), is centralizing the interest of the scientific world. In the next months, long-term consequences on the endocrine system may arise following COVID-19. In this article, we hypothesized the effects of SARS-CoV-2 taking into account what learned from the severe acute respiratory syndrome coronavirus (SARS-CoV) that caused SARS in 2003.
\end{abstract}

Keywords COVID-19 $\cdot$ SARS $\cdot$ Diabetes $\cdot$ Obesity $\cdot$ Adrenal Glands $\cdot$ Pituitary gland

\section{Introduction}

The outbreak of coronavirus disease 2019 (COVID-19) caused by severe acute respiratory syndrome coronavirus 2 (SARS-CoV-2) is centralizing the interest of the scientific world with the aim of understanding its pathogenesis, complications, treatment, and possible long-term consequences.

COVID-19 was first identified in Wuhan [1], the capital city of Hubei province in the People's Republic of China, but then it was declared a Public Health Emergency of International Concern by the World Health Organization (WHO) on January 30, 2020 and on March 11, 2020, COVID-19 was declared a pandemic. According to WHO data (April 25, 2020), 2,864,370 cases of COVID-19 were registered in the world with 199,505 deaths and 816,450 recovered (https://www.worldometers.info/coronavirus/).

Many questions are still not answered about SARS-CoV-2. Various hypotheses have been formulated on its pathogenetic mechanisms and treatment, but very little is known from the endocrinological point of view. In the next months, long-term consequences on the endocrine system may arise in patients

Sandro La Vignera

sandrolavignera@unict.it

1 Department of Clinical and Experimental Medicine, University of Catania, Via S. Sofia 78, 95123 Catania, Italy

2 Department of Experimental and Clinical Medicine, Magna Graecia University Catanzaro, 88100 Catanzaro, Italy who recovered from COVID-19. At moment, we can only hypothesize the effects of SARS-CoV-2 taking into account the knowledge gained on the effects of SARS-CoV, responsible for the 2003 epidemic known as SARS in the Guangdong province of China [2], with 8000 infected patients and 774 deaths [3]. Indeed, SARS-CoV has a genome similar to that of SARS-CoV-2 and shares physiopathological aspects and clinical manifestations [4].

\section{Metabolic aspects}

As for SARS, diabetes mellitus is a high-risk condition for the development of COVID-19 complications and adverse outcomes. Hyperglycemia lowers the immune response thus increasing the risk of mortality $(7.3 \%)$ and it is associated with organ damage and systemic complications [5-7]. In the case of SARS-CoV, it was suggested that the virus could directly damage pancreatic cells, that highly express angiotensinconverting enzyme 2 (ACE2), used as a receptor by the viral spike protein causing acute hyperglycemia [8]. Moreover, SARS-CoV was found in pancreatic tissue by using immunohistochemistry and in-situ hybridization [9].

ACE2 is also involved in SARS-CoV-2 infection since the viral spike protein binds the enzyme and downregulates its expression [10]. According to this observation, we can hypothesize that patients positive to COVID-19 could be subject to virus-mediated pancreatic damage, resulting in the development of diabetes. If this condition occurs, a long-term observation will be needed to understand if 
diabetes will be permanent or SARS-CoV-2 caused a transitory period of hyperglycemia that will resolve with the recovery from the infection.

No study on SARS reported the association between obesity and predisposition to infection or obesity and a higher prevalence of mortality. Many authors generically showed an increase in mortality in patients with comorbidities, but the latter was not defined in detail, so, we can assume that obesity was among them. Some reports have related obesity with worse COVID-19 outcomes and death. Patients with body mass index $(\mathrm{BMI})>35 \mathrm{~kg} / \mathrm{m}^{2}$ have a sevenfold higher risk to receive invasive mechanical ventilation than patients with BMI $<25 \mathrm{~kg} / \mathrm{m}^{2}$ [11]. Moreover, a study conducted in New York, showed that about $21 \%$ of people positive for SARS-CoV-2 were obese, and in patients aged $<60$ years the risk of hospital admission was significantly higher in case of obesity [12]. Obesity and its associated inflammatory state can impair the immune system and it seems to alter the immune response in other models of viral infection, such as influenza [13, 14]. Thus, obesity associated with possible viral direct damage to the pancreatic $\beta$-cells could lead to the development of type 2 diabetes in COVID-19 patients.

\section{Hypothalamic-pituitary-adrenal (HPA) axis}

HPA axis plays a fundamental role in the response to stress. Some studies on SARS suggested that SARS-CoV could impair this hormonal axis by different mechanisms. This virus was found in adrenal and pituitary glands of four patients who died for SARS, so these organs could be the target of infection [9].

In 2004, Wheatland showed that among the strategy used by SARS-CoV to avoid the host's immune response, there was the expression of an amino acid sequence that mimicked human adrenocorticotropic hormone (ACTH). This strategy induced the production of autoantibodies against ACTH, so preventing its action and the suitable adrenal response to stress [15]. Consequently, patients experimented with a condition of relative adrenal insufficiency, characterized by asthenia, myalgia, nausea, anorexia, diarrhea, and headache; these symptoms are common to other viral infections, including influenza virus which shares with SARS-CoV this strategy of the host evasion [15]. Moreover, the interference with ACTH function favors the increase of inflammatory cytokines. Thus, glucocorticoid treatment is a valid therapeutic strategy. According to this observation, the author suggested that an early glucocorticoid administration at low-moderate doses could be preventive, but to avoid the high dosages needed when the treatment was delayed and serious clinical manifestation of SARS developed [15].
Leow et al. explored the function of the HPA axis in 61 SARS survivors [16]. To accomplish that, the authors evaluated serum electrolytes, cortisol, ACTH levels, and 24-h urinary free cortisol. Then, patients with serum cortisol lower than $275 \mathrm{nmol} / 1$ underwent to low dose $(1 \mu \mathrm{g})$, ACTH teststimulation. Adrenal insufficiency was defined for morning cortisol levels below $138 \mathrm{nmol} / \mathrm{l}$ and/or for values $<550 \mathrm{nmol} / 1$ $30 \mathrm{~min}$ after ACTH administration. The authors found that $39.3 \%$ of the patients had hypocortisolism and among them $83.3 \%$ had central adrenal insufficiency [16]. They concluded that adrenal insufficiency could be a late consequence of SARS and it seemed to be secondary to hypophysitis or to direct hypothalamic damage [16].

To date, insufficient data are available on a possible effect of SARS-CoV-2 on the HPA axis function. The concern of scientists is that adrenal insufficiency develops in COVID-19 patients due to a suppression of the axis after glucocorticoid suspension or to exposition to endocrine disruptors that could impair adrenal glands function [17]. Thus, according to the last observations and the knowledge about SARS, adrenal glands function should be assessed in the short-term, to exclude a suppression of the HPA axis in case of glucocorticoid treatment. Moreover, a long-term follow-up will also be necessary to exclude a gradual and late-onset adrenal insufficiency.

\section{Hypothalamic-pituitary-thyroid axis}

Little is known about the effects of SARS-CoV on the thyroid. Ding et al. did not find SARS-CoV expression in thyroid tissue [9], but other authors found central hypothyroidism in SARS survivors, secondary to hypothalamicpituitary dysfunction [16].

Wei et al. reported an impaired thyroid function in patients with SARS, with a decrease in both thyroid hormone and calcitonin levels. Moreover, the thyroid tissue showed an injury of the follicular epithelium and an increase in cell apoptosis, thus suggesting direct tissue damage [18]. A few years later the same group reported decreased serum TSH levels in SARS patients compared with healthy controls. According to this observation, they studied endocrine cells of the pituitary of five patients deceased for SARS, showing a lower number of positive cells and a decreased THS immunoreactive staining [19].

Currently there are no data on possible direct or indirect effects of SARS-CoV-2 on thyroid function, but we think that COVID-19 patients should be monitored for possible changes in thyroid function. Considering that SARS-CoV-2 seems to be able to induce organ damage by autoimmunity [20], we do not exclude also an immune mechanism of thyroid damage. 


\section{Hypothalamic-pituitary-gonadal axis}

No data are reported about SARS and ovarian function. The only evidence available showed an increase in serum levels of prolactin (PRL), follicle-stimulating hormone, and luteinizing hormone and a reduction of 17ß-estradiol (E2) and progesterone levels in SARS patients compared with healthy controls [19]. Moreover, SARS-CoV was not detected in ovarian tissue [9].

Considering that both SARS and COVID-19 are characterized by the possible onset of autoimmunity [20], we believe that the ovarian function of COVID-19 patients should also be monitored.

As for the male reproductive axis, it should be noted that ACE2 is highly expressed by the human testis, which could be infected by SARS-CoV and, probably, by SARS-CoV-2 [10].

Ding et al. did not found SARS-CoV expression in testicular tissue [9]. However, $\mathrm{Xu}$ et al. found tissue damage indicative of orchitis in autopsy testicular tissue obtained from six patients died for SARS. The authors hypothesized that orchitis was a consequence of SARS, suggesting that SARS-CoV could induce testicular damage through an altered immune response [21].

We cannot exclude that testis could be a target of SARSCoV-2 infection, so testicular ultrasound evaluation, hormone assessment, and sperm analysis should be performed in COVID-19 patients in the short- and long-term.

\section{Conclusions}

COVID-19 outbreak is a worldwide issue. There is still much to know on SARS-CoV-2, the etiological agent of this disease. The SARS epidemic in 2003, caused by the SARS$\mathrm{CoV}$, allows us to formulate some hypotheses on the pathogenic mechanisms, but the differences between the two viruses and the enormously higher number of patients affected by COVID19, open unpredictable scenarios. Monitoring the hormone-metabolic function should be implemented by endocrinologists to promptly identify and treat possible long-term pathological conditions.

\section{Compliance with ethical standards}

Conflict of interest The authors declare that they have no conflict of interest.

Publisher's note Springer Nature remains neutral with regard to jurisdictional claims in published maps and institutional affiliations.

\section{References}

1. F. Wu, S. Zhao, B. Yu, Y.M. Chen, W. Wang, Z.G. Song, Y. Hu, Z.W. Tao, J.H. Tian, Y.Y. Pei, M.L. Yuan, Y.L. Zhang, F.H. Dai,
Y. Liu, Q.M. Wang, J.J. Zheng, L. Xu, E.C. Holmes, Y.Z. Zhang, A new coronavirus associated with human respiratory disease in China. Nature 579(7798), 265-269 (2020). https://doi.org/10. 1038/s41586-020-2008-3.

2. J.S. Peiris, S.T. Lai, L.L. Poon, Y. Guan, L.Y. Yam, W. Lim, J. Nicholls, W.K. Yee, W.W. Yan, M.T. Cheung, V.C. Cheng, K.H. Chan, D.N. Tsang, R.W. Yung, T.K. Ng, K.Y. Yuen,SARS study group, Coronavirus as a possible cause of severe acute respiratory syndrome. Lancet. 361(9366), 1319-1325 (2003).

3. Y. Ding, L. He, Q. Zhang, Z. Huang, X. Che, J. Hou, H. Wang, H. Shen, L. Qiu, Z. Li, J. Geng, J. Cai, H. Han, X. Li, W. Kang, D. Weng, P. Liang, S. Jiang, Organ distribution of severe acute respiratory syndrome (SARS) associated coronavirus (SARS-CoV) in SARS patients: implications for pathogenesis and virus transmission pathways. J. Pathol. 203 (2), 622-630 (2004).

4. M. Xie, Q. Chen, Insight into 2019 novel coronavirus-an updated intrim review and lessons from SARS-CoV and MERSCoV. Int. J. Infect. Dis. (2020). S1201-9712(20)30204-6. https:// doi.org/10.1016/j.ijid.2020.03.071.

5. J.W. Chan, C.K. Ng, Y.H. Chan, T.Y. Mok, S. Lee, S.Y. Chu, W. L. Law, M.P. Lee, P.C. Li, Short term outcome and risk factors for adverse clinical outcomes in adults with severe acute respiratory syndrome (SARS). Thorax. 58(8), 686-689 (2003).

6. J.K. Yang, Y. Feng, M.Y. Yuan, S.Y. Yuan, H.J. Fu, B.Y. Wu, G. Z. Sun, G.R. Yang, X.L. Zhang, L. Wang, X. Xu, X.P. Xu, J.C. Chan, Plasma glucose levels and diabetes are independent predictors for mortality and morbidity in patients with SARS. Diabet. Med. 23(6), 623-628 (2006).

7. M. Puig-Domingo, M. Marazuela, A. Giustina, COVID-19 and endocrine diseases. A statement from the European Society of Endocrinology. Endocrine 68(1), 2-5 (2020). https://doi.org/10. 1007/s12020-020-02294-5.

8. J.K. Yang, S.S. Lin, X.J. Ji, L.M. Guo, Binding of SARS coronavirus to its receptor damages islets and causes acute diabetes. Acta Diabetol. 47(3), 193-199 (2010). https://doi.org/10.1007/ s00592-009-0109-4.

9. Y. Ding, L. He, Q. Zhang, Z. Huang, X. Che, J. Hou, H. Wang, H. Shen, L. Qiu, Z. Li, J. Geng, J. Cai, H. Han, X. Li, W. Kang, D. Weng, P. Liang, S. Jiang, Organ distribution of severe acute respiratory syndrome (SARS) associated coronavirus (SARS$\mathrm{CoV}$ ) in SARS patients: implications for pathogenesis and virus transmission pathways. J Pathol. 203(2), 622-630 (2004).

10. S. La Vignera, R. Cannarella, R.A. Condorelli, F. Torre, A. Aversa, A.E. Calogero, Sex-specific SARS-CoV-2 mortality: among hormone-modulated ACE2 expression, risk of venous thromboembolism and hypovitaminosis D. Int. J. Mol. Sci. 21(8), E2948 (2020). https://doi.org/10.3390/ijms21082948.

11. N. Sattar, I.B. McInnes, J.J.V. McMurray, Obesity a risk factor for severe COVID-19 infection: multiple potential mechanisms. Circulation. (2020). https://doi.org/10.1161/CIRCULATIONAHA. 120.047659 .

12. J. Lighter, M. Phillips, S. Hochman, S. Sterling, D. Johnson, F. Francois, A. Stachel, Obesity in patients younger than 60 years is a risk factor for Covid-19 hospital admission. Clin. Infect. Dis. ciaa415 (2020). https://doi.org/10.1093/cid/ciaa415.

13. F.P. de Heredia, S. Gómez-Martínez, A. Marcos, Obesity, inflammation and the immune system. Proc. Nutr. Soc. 71(2), 332-338 (2012). https://doi.org/10.1017/S0029665112000092.

14. W.D. Green, M.A. Beck, Obesity impairs the adaptive immune response to influenza virus. Ann. Am. Thorac. Soc. 14(Supplement_5), S406-S409 (2017). https://doi.org/10.1513/AnnalsATS. 201706-447AW.

15. R. Wheatland, Molecular mimicry of ACTH in SARS-implications for corticosteroid treatment and prophylaxis. Med. Hypotheses. 63(5), 855-862 (2004). 
16. M.K. Leow, D.S. Kwek, A.W. Ng, K.C. Ong, G.J. Kaw, L.S. Lee, Hypocortisolism in survivors of severe acute respiratory syndrome (SARS). Clin. Endocrinol. 63(2), 197-202 (2005).

17. C. Scaroni, M. Armigliato, S. Cannavò, COVID-19 outbreak and steroids administration: are patients treated for Sars-Cov-2 at risk of adrenal insufficiency? J. Endocrinol. Investig. (2020). https:// doi.org/10.1007/s40618-020-01253-1.

18. L. Wei, S. Sun, C.H. Xu, J. Zhang, Y. Xu, H. Zhu, S.C. Peh, C. Korteweg, M.A. McNutt, J. Gu, Pathology of the thyroid in severe acute respiratory syndrome. Hum. Pathol. 38(1), 95-102 (2007).
19. L. Wei, S. Sun, J. Zhang, H. Zhu, Y. Xu, Q. Ma, M.A. McNutt, C. Korteweg, J. Gu, Endocrine cells of the adenohypophysis in severe acute respiratory syndrome (SARS). Biochem. Cell Biol. 88(4), 723-730 (2010). https://doi.org/10.1139/O10-022.

20. J. Lyons-Weiler, Pathogenic priming likely contributes to serious and critical illness and mortality in COVID-19 via autoimmunity. J. Transl. Autoimmun. 100051 (2020). https://doi.org/10.1016/j. jtauto.2020.100051.

21. J. Xu, L. Qi, X. Chi, J. Yang, X. Wei, E. Gong, S. Peh, J. Gu, Orchitis: a complication of severe acute respiratory syndrome (SARS). Biol. Reprod. 74(2), 410-416 (2006). 